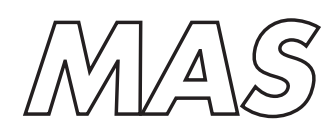

Modelling, Analysis and Simulation

Modelling, Analysis and Simulation
MAS A finite-volume method for convection problems with
embedded moving boundaries

Y.J. Hassen, B. Koren

RePORt MAS-E0901 JanUARY 2009 
Centrum Wiskunde \& Informatica (CWI) is the national research institute for Mathematics and Computer Science. It is sponsored by the Netherlands Organisation for Scientific Research (NWO).

$\mathrm{CWI}$ is a founding member of ERCIM, the European Research Consortium for Informatics and Mathematics.

CWI's research has a theme-oriented structure and is grouped into four clusters. Listed below are the names of the clusters and in parentheses their acronyms.

Probability, Networks and Algorithms (PNA)

Software Engineering (SEN)

\section{Modelling, Analysis and Simulation (MAS)}

Information Systems (INS)

Copyright (C) 2009, Centrum Wiskunde \& Informatica

P.O. Box 94079, 1090 GB Amsterdam (NL)

Science Park 123, 1098 XG Amsterdam (NL)

Telephone +31205929333

Telefax +31 205924199 


\title{
A finite-volume method for convection problems with embedded moving boundaries
}

\author{
ABSTRACT \\ An accurate method, using a novel immersed-boundary approach, is presented for numerically \\ solving linear, scalar convection problems. Moving interior boundary conditions are embedded \\ in the fixed-grid fluxes in the direct neighborhood of the moving boundaries. Tailor-made limiters \\ are derived such that the resulting scheme is monotone. The results obtained are very accurate, \\ without requiring much computational overhead. It is anticipated that the method can readily be \\ extended to real fluid-flow equations.
}

2000 Mathematics Subject Classification: 65M15 65M20 65M60

Keywords and Phrases: immersed-boundary method; hyperbolic conservation laws; high-order schemes; monotonicity; limiters; time adaptivity.

Note: The first author's research is funded by the Delft Centre for Computational Science and Engineering (DCSE), TU Delft. 



\title{
A Finite-Volume Method for Convection Problems with Embedded Moving-Boundaries
}

\author{
Yunus Hassen ${ }^{1,2}$ and Barry Koren ${ }^{1,3}$ \\ 1 Centrum Wiskunde \& Informatica, Amsterdam, the Netherlands \\ 2 Faculty of Aerospace Engineering, TU Delft, the Netherlands \\ 3 Mathematical Institute, Leiden University, the Netherlands \\ yunus.hassen@cwi.nl, barry.koren@cwi.nl
}

Summary. An accurate method, using a novel immersed-boundary approach, is presented for numerically solving linear, scalar convection problems. Moving interior boundary conditions are embedded in the fixed-grid fluxes in the direct neighborhood of the moving boundaries. Tailor-made limiters are derived such that the resulting scheme is monotone. The results obtained are very accurate, without requiring much computational overhead. It is anticipated that the method can readily be extended to real fluid-flow equations.

Key words: immersed-boundary method; hyperbolic conservation laws; high-order schemes; monotonicity; limiters; time adaptivity.

\section{Introduction}

The immersed-boundary method, in general, is a method in which boundary conditions are indirectly incorporated into the governing equations. It has first been introduced by Peskin [4], and currently many varieties of it exist.

Immersed-boundary methods are very suitable for simulating flows around flexible, moving and/or complex bodies. Basically, the bodies of interest are just embedded in non-deforming Cartesian grids that do not conform to the shape of the body. The governing equations are modified to include the effect of the embedded bodies (EBs). Doing so, mesh (re)generation difficulties associated with body-fitted grids are obviated; and, the underlying regular fixed grid allows to use a simple data structure as well as simpler numerical schemes over a majority of the domain.

Our approach uses a cell-centered finite-volume discretization. The governing partial differential equations are discretized using a standard finite-volume method (FVM) away from the EBs. Near the EB, a special FVM is derived which takes the prescribed interior boundary conditions into account.

The article begins with the problem description and with some standard finitevolume results. The following sections present: the special fluxes that take the effects of the EBs into account, the temporal discretization, monotonicity domains and 
limiters, and time adaptivity, in the respective order. Finally, some numerical results, based on the present approach, and concluding remarks are given.

\section{Model equation}

Consider the scalar, linear convection equation:

$$
\frac{\partial c}{\partial t}+\frac{\partial f}{\partial x}=0, \quad f=f(c):=u c,
$$

where $c(x, t)$ is the scalar field, $u$ the flow velocity, which is assumed to be constant and positive, and $f(c)$ the flux function. The independent variables $x$ and $t$ represent space and time, respectively. We take $x \in[0,1]$.

Eq. (1) is hyperbolic. The initial solution $c(x, 0)=c_{0}(x)$ simply propagates unchanged with the velocity $u: c(x, t):=c_{0}(x-u t)$. We consider two initial solutions, each with two interior, moving EBs. The solution at the left and right of each EB is prescribed. The two moving EBs have arbitrary initial locations $\left(0 \leq x_{1} \leq 1\right.$ and $\left.0 \leq x_{2} \leq 1, x_{1} \neq x_{2}\right)$. The initial solutions read:

$$
c_{0}(x)=\left\{\begin{array}{ll}
0, & \text { if } x_{1} \leq x \leq x_{2}, \\
1, & \text { elsewhere }
\end{array} \quad \text { and } \quad c_{0}(x)= \begin{cases}0, & \text { if } x_{1} \leq x \leq x_{2}, \\
\frac{1-\cos (2 \pi x)}{2}, & \text { elsewhere }\end{cases}\right.
$$

The cosine function in (2) exploits the advantage that higher-order accurate numerical schemes have in non-constant, smooth solution regions. Model equation (1) is approximated in a periodic domain, allowing us to time-step for as long as we want for a given, finite, spatial domain.

\subsection{Standard FVM results}

The unit domain is divided into $N$ non-overlapping cells of uniform size. Let $h=1 / N$ be the cell width, $x_{i}=(i-1 / 2) h$ the cell-center coordinates and $x_{i+\frac{1}{2}}=i h$ the cell-face coordinates for $i=1,2, \ldots, N$. Let the fully discrete solution in cell $i$, at time level $n$, be denoted as $c_{i}^{n}=c\left(x_{i}, t^{n}\right)$. Then the semi-discrete finite-volume form of (1) reads:

$$
h \frac{\mathrm{d} c_{i}}{\mathrm{~d} t}+\left\{\left(f_{i+\frac{1}{2}}(t)-f_{i-\frac{1}{2}}(t)\right\}=0 .\right.
$$

Eq. (3) is exact, inside a specific cell, so far and it is solved by approximating the fluxes, at time level $n$, say $f_{i+\frac{1}{2}}^{n}$, and by time-stepping the temporal part. The fluxes are computed (dropping the index $n$, for convenience) as $f_{i+\frac{1}{2}}=u c_{i+\frac{1}{2}}$, where $c_{i+\frac{1}{2}}$ is the cell-face state at $i+\frac{1}{2}$, which can be approximated in a variety of ways. For example, for $u>0, c_{i+\frac{1}{2}}=c_{i}$ and $c_{i+\frac{1}{2}}=c_{i}+\frac{1+\kappa}{4}\left(c_{i+1}-c_{i}\right)+\frac{1-\kappa}{4}\left(c_{i}-c_{i-1}\right)$ are two classical cell-face states, computed with the first-order upwind- and van Leer's $\kappa$-scheme [6], respectively. Note that, with no EB in the neighborhood, $\kappa \in[-1,1]$.

The $\kappa$-schemes yield non-monotone discretizations. Several algorithms have been proposed in the literature that yield higher-order accurate, monotone solutions. Most of these algorithms exploit the inherent monotonicity of the first-order upwind scheme. The best known representatives of these algorithms are the limited schemes following Sweby's total-variation diminishing (TVD) theory [5]. 
The cell-face state $c_{i+\frac{1}{2}}$ can be written in the limited form as $c_{i+\frac{1}{2}}=c_{i}+$ $\frac{1}{2} \phi\left(r_{i+\frac{1}{2}}\right)\left(c_{i}-c_{i-1}\right)$, where $\phi(r)$ is the limiter function and $r_{i+\frac{1}{2}}=\frac{c_{i+1}-c_{i}}{c_{i}-c_{i-1}}$ its monotonicity argument. Here we specifically adopt the limiter proposed by Koren [3] as the standard limiter. It gives a monotone third-order accurate net flux in a cell, by resembling the $\kappa=\frac{1}{3}$-scheme.

Now, for later comparison purposes, we will show what the solutions are when using the standard finite-volume discretizations described above, methods in which no embedded-boundary conditions are imposed. For the time integration, the threestage Runge-Kutta scheme RK3b from [2] is employed. For both initial solutions (2), we consider the locations of the EBs to be at $x_{1}=\frac{1}{3}$ and $x_{2}=\frac{2}{3}$. Furthermore, we take $u=1$, and we compute the solution at $t=1$, the time at which the solution has made a single full-period. For both the first-order upwind and the $\kappa=\frac{1}{3}$ (unlimited and limited) schemes, the computations are performed on a grid with 20 and 40 cells. The solutions are depicted in Fig. 1. The time steps have been taken sufficiently small to ensure that in all cases the temporal discretization errors are negligible with respect to the spatial discretization errors.

\section{Fluxes with embedded moving-boundary conditions}

The sharp discontinuities of the initial solutions (2) are considered as infinitely thin bodies going with the flow and the boundary conditions associated with these are
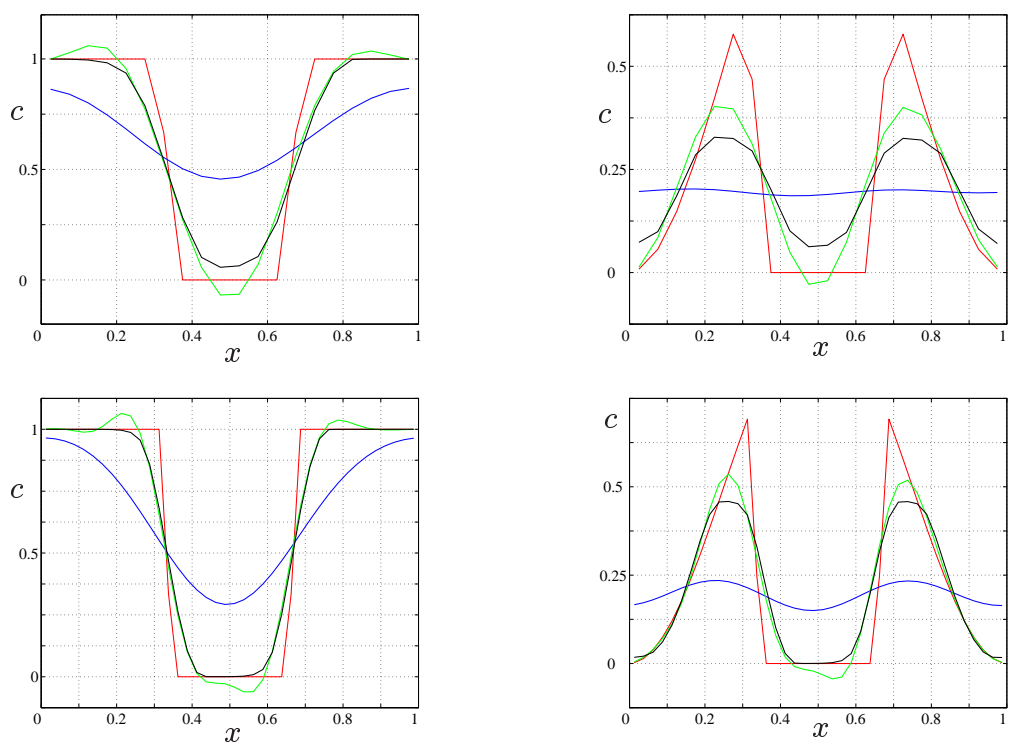

Fig. 1. Standard finite-volume solutions after one full-period. Red: exact discrete, blue: first-order upwind, green: unlimited $\kappa=\frac{1}{3}$ scheme, and black: limited ditto. Note: the top two are the results on a 20-cell grid and the bottom two are on a 40-cell grid. 
embedded in the fixed-grid fluxes. Here, the embedded-boundary conditions are user-specified and enforced to remain intact to the EB and unchanged at all times. The solution values on the left and right sides of the EB are designated as $c_{\mathrm{EB}}^{l}$ and $c_{\mathrm{EB}}^{r}$, respectively (Fig. 2).

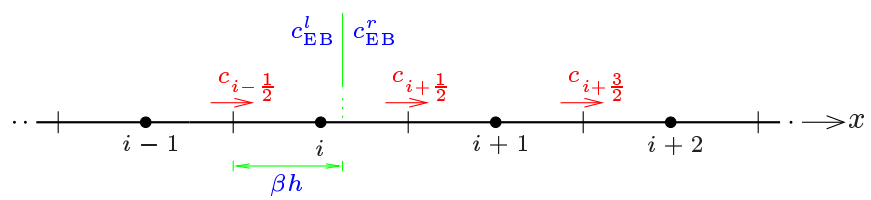

Fig. 2. EB situated in cell $i$ at time $t^{n}$, its associated solution values (to be used as 'embedded-boundary conditions'), and the three affected cell-face states.

For an EB situated in cell $i$, with its coordinate $x_{\mathrm{EB}}(t)=x_{\mathrm{EB}}^{n}$ given, its relative position with respect to the cell face $x_{i-\frac{1}{2}}$ is $\beta h$, where:

$$
\beta=\frac{x_{\mathrm{EB}}^{n}-x_{i-\frac{1}{2}}}{h}, \quad \beta \in[0,1] .
$$

There is no information flow across the EB. Fluxes on one side of the EB are all computed based on the information on the same side and the additional interior boundary condition on the respective side of the EB. In general, when considering three-point upwind-biased interpolation for the fluxes, three cell-face states $\left(c_{i-\frac{1}{2}}\right.$, $c_{i+\frac{1}{2}}$ and $c_{i+\frac{3}{2}}$ ) are affected by the presence of a single EB (in cell $i$ ) and these are the cell-face states of interest that are especially modified (Fig. 2). $c_{i-\frac{1}{2}}$ and $c_{i+\frac{3}{2}}$ are written as optimally blended, three-point upwind-biased interpolation formulae:

$$
\begin{aligned}
c_{i-\frac{1}{2}} & =c_{i-1}+\frac{1}{1+2 \beta} \frac{1+\kappa_{i-\frac{1}{2}}}{2}\left(c_{\mathrm{EB}}^{l}-c_{i-1}\right)+\frac{1-\kappa_{i-\frac{1}{2}}}{4}\left(c_{i-1}-c_{i-2}\right), \\
c_{i+\frac{3}{2}} & =c_{i+1}+\frac{1+\kappa_{i+\frac{3}{2}}}{4}\left(c_{i+2}-c_{i+1}\right)+\frac{2}{3-2 \beta} \frac{1-\kappa_{i+\frac{3}{2}}}{4}\left(c_{i+1}-c_{\mathrm{EB}}^{r}\right) .
\end{aligned}
$$

Since we do not draw information across the EB, no upwind-biased interpolation formula can be derived for $c_{i+\frac{1}{2}}$. Non-equidistant central interpolation is applied to compute $c_{i+\frac{1}{2}}$.

The blending parameters $\kappa_{i-\frac{1}{2}}$ and $\kappa_{i+\frac{3}{2}}$ are optimized such that the net fluxes in cells $i-1$ and $i+2$, respectively, are as accurate as possible. The net flux in cell $i$ cannot be optimized due to the presence of the EB with its discontinuous solution behavior. Deriving the modified equations in cells $i-1$ and $i+2$, and equating the leading term of the truncation errors to zero, we get:

$$
\kappa_{i-\frac{1}{2}}=\frac{7-6 \beta}{9+6 \beta}, \quad \kappa_{i-\frac{1}{2}} \in\left[\frac{1}{15}, \frac{7}{9}\right] \quad \text { and } \quad \kappa_{i+\frac{3}{2}}=\frac{7-6 \beta}{15-6 \beta}, \quad \kappa_{i+\frac{3}{2}} \in\left[\frac{1}{9}, \frac{7}{15}\right] .
$$

The reasons to consider the net flux in cell $i+2$ instead of that of cell $i+1$, for optimizing $\kappa_{i+\frac{3}{2}}$, are given in [1]. The formulae for the EB-affected cell-face states are summarized, in terms of the parameter $\beta$, as: 


$$
\begin{aligned}
& c_{i-\frac{1}{2}}=c_{i-1}+\frac{8}{(3+6 \beta)(3+2 \beta)}\left(c_{\mathrm{EB}}^{l}-c_{i-1}\right)+\frac{1+6 \beta}{18+12 \beta}\left(c_{i-1}-c_{i-2}\right), \\
& c_{i+\frac{1}{2}}=c_{\mathrm{EB}}^{r}+\frac{2-2 \beta}{3-2 \beta}\left(c_{i+1}-c_{\mathrm{EB}}^{r}\right), \\
& c_{i+\frac{3}{2}}=c_{i+1}+\frac{11-6 \beta}{30-12 \beta}\left(c_{i+2}-c_{i+1}\right)+\frac{4}{(9-6 \beta)(5-2 \beta)}\left(c_{i+1}-c_{\mathrm{EB}}^{r}\right) .
\end{aligned}
$$

Note that it is assumed that two successive EBs are sufficiently far apart, such that a given cell-face state is affected by only one EB. Recall that all but the EB-affected fluxes are computed with a standard scheme.

\section{Temporal discretization}

After substituting the appropriate discretizations for the spatial operator in the semi-discrete equation (3), it is integrated in time using an explicit method: either the Forward Euler or the RK3b [2] scheme. The later gives a third-order accuracy in time.

\subsection{Monotonicity and limiters}

Noting that the EB-affected cell-face states (7) are higher-order accurate and linear, wiggles are imminent. These wiggles can be suppressed by carefully constraining the convective cell-face states. Therefore, as explained in detail in [1], we define nonstandard monotonicity arguments, $\tilde{r}_{i-\frac{1}{2}}$ and $\tilde{r}_{i+\frac{3}{2}}$, and derive the limited forms of $c_{i-\frac{1}{2}}$ and $c_{i+\frac{3}{2}}$. The cell-face state $c_{i+\frac{1}{2}}$, however, is not limited as we can not define a monotonicity argument $\tilde{r}_{i+\frac{1}{2}}$. Enforcing appropriate monotonicity requirements, the resulting EB-sensitive limiter-functions $\tilde{\phi}(\tilde{r})$ become $\nu$-dependent (where $\nu=u \tau / h$ is the CFL number and $\tau$ is the time step). The $\nu$-dependence, however, is avoided by taking a stringent restriction $\nu \leq \frac{1}{2}$, to achieve a monotonicity preserving scheme and a second-order accurate discrete-solution. Then, the resulting bounds for $\tilde{\phi}(\tilde{r})$ are simplified and they are fully constrained, $\forall \tilde{r}_{i-\frac{1}{2}}$ and $\forall \tilde{r}_{i+\frac{3}{2}}$, as:

$$
\begin{gathered}
0 \leq \tilde{\phi}\left(\tilde{r}_{i-\frac{1}{2}}\right) \leq 2 \quad \text { and } \quad \frac{\tilde{\phi}\left(\tilde{r}_{i-\frac{1}{2}}\right)}{\tilde{r}_{i-\frac{1}{2}}} \leq 1+2 \beta, \\
-1 \leq \tilde{\phi}\left(\tilde{r}_{i+\frac{3}{2}}\right) \leq 5-4 \beta \quad \text { and } \quad 0 \leq \frac{\tilde{\phi}\left(\tilde{r}_{i+\frac{3}{2}}\right)}{\tilde{r}_{i+\frac{3}{2}}} \leq 2,
\end{gathered}
$$

Typical limiters, satisfying the special bounds (8), are depicted in Fig. 3.

\subsection{Local adaptivity in time}

If an EB is situated in such a way that $x_{\mathrm{EB}}^{n} \in\left[x_{i-\frac{1}{2}}, x_{i+\frac{1}{2}}\right)$ and $x_{\mathrm{EB}}^{n+1} \in\left[x_{i+\frac{1}{2}}, x_{i+\frac{3}{2}}\right)$, there is an abrupt change in $c_{i+\frac{1}{2}}$ when going from $t^{n}$ to $t^{n+1}$ (see Fig. 4). To account for this change, time adaptivity is introduced by first computing the time fraction $\alpha$ at which the EB crosses $x_{i+\frac{1}{2}}$, as: 

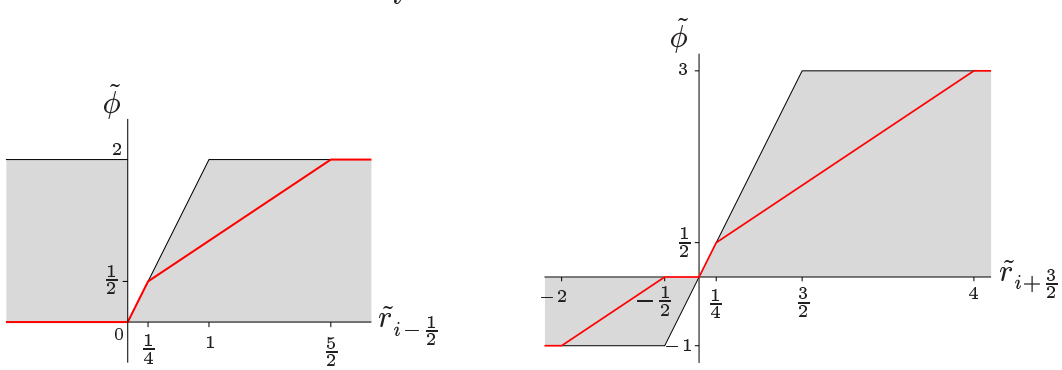

Fig. 3. Typical EB-sensitive limiters (for $\beta=1 / 2$ ), within the corresponding, simplified, monotonicity domains, for the EB-affected cell-face states $c_{i-\frac{1}{2}}$ (left) and $c_{i+\frac{3}{2}}$ (right).

$$
\alpha=\frac{x_{i+\frac{1}{2}}+\epsilon-x_{\mathrm{EB}}^{n}}{u \tau}, \quad \alpha \in(0,1) .
$$

Next, the intermediate cell-face state $c_{i+\frac{1}{2}}^{n+\alpha}$ is computed. Note that the EB is placed at infinitesimal distance $\epsilon$ off $x_{i+\frac{1}{2}}$, in the direction of the flow. Then the cell-face state $c_{i+\frac{1}{2}}^{n}$ is recomputed as the weighted average:

$$
c_{i+\frac{1}{2}}^{n}:=\alpha c_{i+\frac{1}{2}}^{n}+(1-\alpha) c_{i+\frac{1}{2}}^{n+\alpha} .
$$

Finally, solution updating, in Forward Euler, is continued everywhere, using the time-adapted cell-face state, with the regular time step $\tau$. For RK3b, we do not yet resort to the temporal local-adaptivity. We instead split the regular time step $\tau$ into smaller time steps, depending on the number of EBs crossing cell faces, and update the intermediate solutions everywhere.

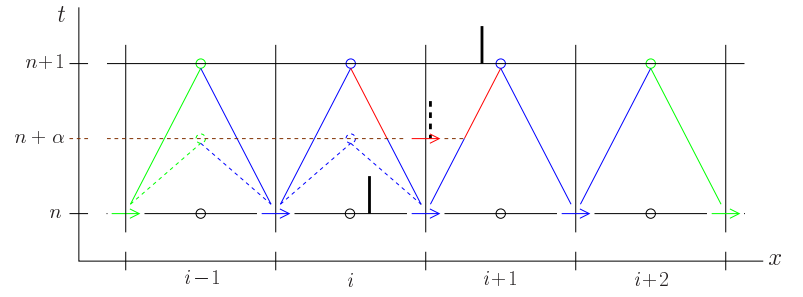

Fig. 4. Stencil for local adaptivity in time. The standard, modified and the intermediate cell-face fluxes are designated in green, blue, and red, respectively.

\section{Results and conclusion}

We present numerical results to validate the immersed-boundary approach introduced in this work. We take the same data as in $\S 2.1$. The results obtained, shown in Fig. 5, are remarkably accurate. They show a significant improvement in resolution over those computed using the standard methods (Fig. 1). For the more 
discriminating initial solution, the cosine-cavity in (2), the numerical results of the limited higher-order upwind-biased schemes are slightly deficient at the peripheries. This is due to the property of limiters that they clip physically relevant extrema. Apparently, the deficiency diminishes with decreasing grid size.
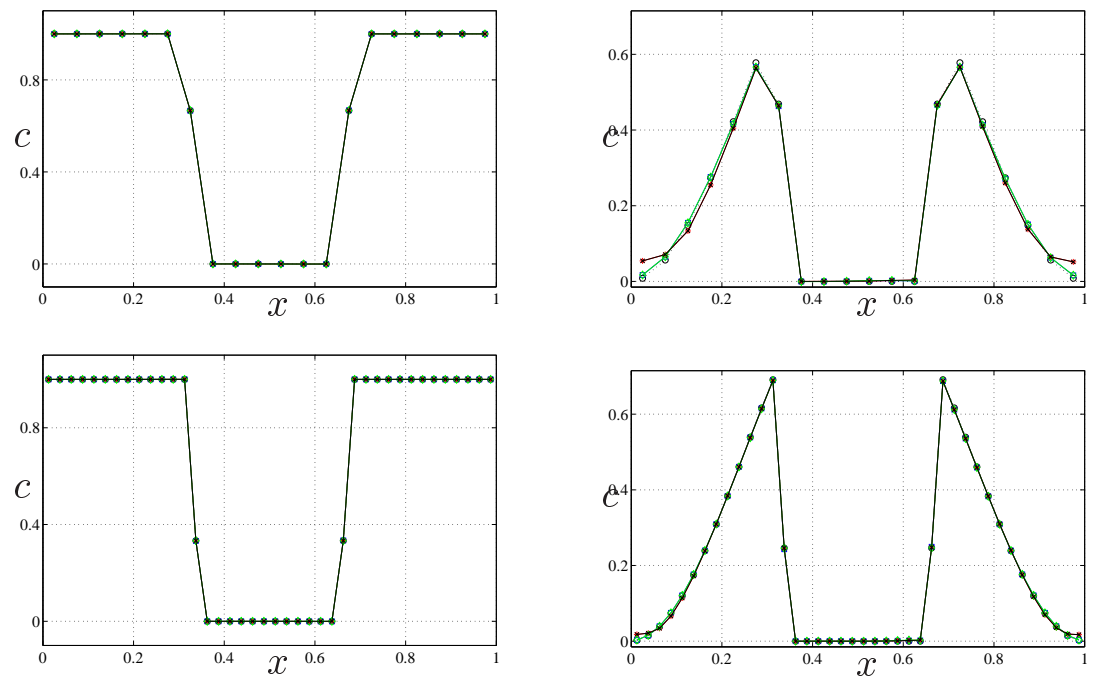

Fig. 5. Immersed-boundary solutions after one full-period. $\bigcirc$ : exact discrete, $\square$ : unlimited higher-order upwind-biased with Forward Euler, $*$ : limited ditto, $\diamond$ : unlimited higher-order upwind-biased with RK3b, $\times$ : limited ditto. Note: the top two are the results on a 20 -cell grid and the bottom two are on a 40 -cell grid.

The essence of the present approach is that moving bodies are embedded in a regular fixed grid and specific fluxes in the vicinity of the embedded boundary are intelligently computed in such a way that they accurately accommodate the boundary conditions valid on the moving EB. Then, over the majority of the domain, where we do not have influence of the EBs, we use standard methods on the underlying regular fixed grid. Excellent results are achieved, without much computational overhead. We foresee that the numerical methods introduced here can readily be extended to real fluid-flow equations.

\section{References}

1. Hassen, Y., Koren, B.: In LNCSE. Springer (To appear in 2009).

2. Hundsdorfer, W. et al.: J. Comput. Phys. 117, 35-46 (1995).

3. Koren, B.: In Notes on Num. Fl. Mech., 45, 117-138. Vieweg (1993).

4. Peskin, C.S.: J. Comput. Phys. 10, 252-271 (1972).

5. Sweby, P.: SIAM J. Num. Anal. 21, 995-1011 (1984).

6. van Leer, B.: In Lect. Appl. Math., 22-2, 327-336. AMS, RI (1985). 